\title{
Intrafollicular oocyte transfer in cattle - a technical report
}

\author{
Michaela Andrlikova ${ }^{1}$, Vladislav Bina ${ }^{2}$, Vojtech $\operatorname{Kos}^{1}$, Miloslava Lopatarova ${ }^{1}$, \\ Beata Markova ${ }^{1}$, Lucie Stenclova ${ }^{1}$, Svatopluk Cech $^{1}$ \\ ${ }^{1}$ University of Veterinary and Pharmaceutical Sciences Brno, Faculty of Veterinary Medicine, \\ Ruminant and Swine Clinic, Brno, Czech Republic \\ ${ }^{2}$ University of Economics Prague, Faculty of Management, Department of Exact Methods, Prague, Czech Republic
}

Received November 13, 2019

Accepted January 28, 2020

\begin{abstract}
The aim of the study was to develop and evaluate the functionality of a new equipment for intrafollicular oocyte transfer (IFOT) in dairy cattle. The new system for IFOT is composed of the applicator, the aspirator, and the injector. After aspiration of oocytes, the IFOT set is inserted into the working tube of the ultrasound transducer holder, the content of the applicator can be injected into the preovulatory follicle via transvaginal ultrasonography by one operator. The function of instruments used for IFOT was firstly verified in laboratory conditions. Slaughterhouse oocytes filled into the instruments were injected into Petri dishes. The highest recovery rates in vitro $(97.5 \%)$ were achieved when the applicator was stored with the needle in a downward position. Synchronized Holstein heifers were used for in vivo test. Intrafollicular injection of saline $(\mathrm{n}=9)$ was performed to find whether ovulation is affected by the injection. Then IFOTs of phosphate buffered saline with 20 oocytes $(n=21)$ were performed into the preovulatory follicles followed by 7 -day-old embryo collection. Total ovulation rates were $86.7 \%(26 / 30)$. Total recovery rates (oocytes + embryos) were $23.1 \%$, embryo recovery rates were $10.1 \%$. The new instrument allows for the loading of oocytes and easy transportation to recipients, and also allows IFOT to be performed by one person in field conditions. The method does, however, need further investigation.
\end{abstract}

Heifer, in vitro embryo production, ovarian transvaginal ultrasonography, intraovarian injection

Different assisted reproductive techniques (ART) have been developed and used in human medicine, animal husbandry and wildlife management. Embryo technologies including embryos derived in vivo (IVD) as well as in vitro (IVP) are widely used in cattle to improve the genetic merit of population (Findlay et al. 2019). Embryo production in vitro is a very important tool for reducing the generation interval by using oocytes from very young or even prepubertal donors (Kasinathan et al. 2015). However, IVP embryos deviate from its in vivo counterparts as impaired developmental competence, lipid content, ultrastructure, cryotolerance and gene expression may occur (Lonergan and Fair 2008; O'Doherty et al. 2018). Furthermore, oocytes recovered from young females are qualitatively inferior to oocytes recovered from adult animals (Landry et al. 2016). The development of new in vitro culture conditions steadily results in increasing numbers of embryos from IVP (Moreno et al. 2015). Nevertheless, the in vitro culture conditions fail to completely mimic the physiological environment. The impact of in vivo culture on the quality of bovine embryos in bovine oviduct has been shown (Besenfelder et al. 2010).

Recently, a new method called intrafollicular oocyte transfer (IFOT) has been developed (Kassens et al. 2015). This method is based on injection of in vitro collected oocytes into the preovulatory follicle of recipient heifers that are subsequently inseminated. Consequently, maturation, ovulation, fertilization and early embryo development take place in the natural environment in the follicle, oviduct and the uterus of the recipient, respectively. At Day 7 embryos are recovered by routine uterine flushing. Although

Address for correspondence:

doc. MVDr. Svatopluk Cech, Ph.D.

Ruminant and Swine Clinic, Faculty of Veterinary Medicine

University of Veterinary and Pharmaceutical Sciences Brno

Palackého tř. 1946/1, 61242 Brno, Czech Republic 
numbers of embryos obtained were highly variable, it was shown that IFOT of in vitro matured oocytes represents a feasible technique for generating bovine blastocysts closely resembling IVD blastocysts in terms of lipid content and cryotolerance (Kassens et al. 2015). In the following study, immature oocytes were transferred into growing follicles prior to ovulation. This study demonstrated that even in vitro maturation can be bypassed by direct transfer of collection cumulus-oocytes complexes into the follicle (Hoelker et al. 2017). Intrafollicular oocyte transfer using immature fresh or vitrified oocytes collected from living donors using transvaginal aspiration was reported (Sprícigo et al. 2016). Although IFOT did not improve the developmental competence of vitrified oocytes, viable blastocysts and pregnancies were achieved after transfer of fresh immature oocytes. This method is an option for clinical bovine embryo production without in vitro culture (Sprícigo et al. 2016).

From a technical point of view, IFOT is based on ultrasound-guided transvaginal follicular aspiration (TVFA), which was first reported more than thirty years ago. The first application of TVFA was oocyte aspiration (ovum pick up, OPU) (Pieterse et al. 1988). However, TVFA has been permanently modified and improved to increase feasibility and practical application. The collective term ovarian transvaginal ultrasonography (OTU) has been used to denote the entire OPU system (Velazquez et al. 2014). Since oocyte collection and oocyte transfer do not differ substantially, OPU systems have been slightly modified in order to perform first studies on IFOT. Equipment described in all mentioned IFOT studies (Kassens et al. 2015; Sprícigo et al. 2016; Hoelker et al. 2017) consists of a plastic holder and an ultrasound transducer $(7.5-10 \mathrm{MHz})$.

A long tubing system was connected with a disposable needle (G26, G27) at one end and with either a laboratory pipette or a $1 \mathrm{ml}$ syringe at the other end. The tubing system was filled with fluid (phosphate-buffered saline or follicular fluid). Oocytes were loaded into the needle at a fluid volume of 60 or $200 \mu 1$ (Kassens et al. 2015; Sprícigo et al. 2016; Hoelker et al. 2017). Handling required at least two people: one person performed rectal manipulation and navigated the follicle bearing ovary in front of the ultrasound; the second person introduced IFOT set and took care of the careful oocyte transfer into the follicle.

The aim of our study was to develop a new application set for IFOT enabling: 1) aspiration of in vitro collected oocytes into the transfer system; 2) transport of the transfer system to the place where recipient animals will be prepared; 3) intrafollicular oocyte transfer by only one operator under field conditions.

\section{IFOT set}

\section{Materials and Methods}

The IFOT set consisted of three sub-units: applicator, aspirator, and injector. The applicator is composed of a 1-ml syringe connected to a disposable needle $(25 \mathrm{G}, 0.55 \times 40 \mathrm{~mm}$, Braun, Germany). In order to ensure that oocyte transfer via the needle into the follicle will be successful, a precisely manufactured stainless steel adapter was developed to cover a piece of a $0.25 \mathrm{ml}$ insemination straw that was inserted between needle and syringe to minimize dead space where oocytes could remain in the applicator system (Plate I, Fig. 1, Fig. 2). The size of the piece of insemination straw was designed to match the volume of aspirated COC medium containing oocytes. The applicator served three functions: the loading of oocytes, transportation in a thermo-box to animals, and intra-follicular oocyte transfer.

The aspirator was custom-made, comprising an iron screw with a fine thread to precisely move the plunger of the syringe (Plate I, Fig. 3). The aspirator allows the careful loading of the oocytes via the needle into the piece of plastic straw and easy transportation to recipients (Plate I, Fig. 4, Plate II, Fig. 5).

The injector is a $50 \mathrm{~cm}$ long instrument equipped with an affixation point for the insertion of the applicator at the front part, a pulling rod running through the entire tool, and a guide pin at the back part of the injector (Plate II, Fig. 6.). The syringe is attached at the affixation point whereas the syringe plunger is fixed to the front part of the pulling rod. When the plastic holder was inserted into the vagina and its tip reached the middorsal area closed to the fornix, the whole injector was carefully inserted into the guide tube of the plastic holder (Plate II, Fig. 7). After the follicle was ultrasonographically (Kaixin TM-5200V, Xuzhou, China, a micro-convex array 7.5 MHz transducer) presented in front of the holder, the guide pin was moved by the operator outside of the animal and the content of the applicator was injected into the preovulatory follicle under visual control (Plate III, Fig. 8.). 
Experiment 1: Feasibility test of the IFOT instruments under laboratory conditions Oocyte preparation

Bovine cumulus-oocyte complexes (COCs) derived from abattoir ovaries were used in this study. The ovaries were transported to the lab in tempered saline at $30{ }^{\circ} \mathrm{C}$ in a thermobox within $3 \mathrm{~h}$ of collection. The COCs were obtained by follicle slicing and selected for only those that were surrounded by at least 3 layers of cumulus cells. The COCs were cultured in 4-well dishes in a standard maturation medium covered with paraffin oil at $39{ }^{\circ} \mathrm{C}$ in a humidified atmosphere with $5 \% \mathrm{CO}_{2}$ for $16-22 \mathrm{~h}$.

\section{Technical validation of the IFOT system}

Phosphate-buffered saline (PBS) was used for manipulation of matured COCs. First PBS (100 $\mu 1)$ was loaded into the applicator by manipulation of the screw of the aspirator, then 10-20 $\mu$ of PBS with 10 COCs were aspirated with the needle and placed in the front part of the cut plastic straw. The applicator was removed from the aspirator and fit in the tip of the injector. Moving the guide pin and pulling rod resulted in the release of PBS plus COCs into the Petri dish. The number of recovered COCs was assessed under the stereomicroscope.

Two factors were evaluated:

1. Two lengths of the cut plastic straw $(15 \mathrm{~mm}$ and $18 \mathrm{~mm})$ were tested. The $15 \mathrm{~mm}$ length originated by cutting the straw exactly at the front end of the adapter, the straw did not protrude from the adapter to the Luer-Lock fitting of the needle. The $18 \mathrm{~mm}$ length protruded from the adapter and exactly filled the whole Luer-Lock fitting of the needle without any dead space.

2. The applicators containing oocytes were positioned in a thermobox for $3 \mathrm{~min}$ in three different positions to determine if the sedimentation of the oocytes affected the recovery rate. The needle was positioned downwards in group 1, upwards in group 2 and applicators were left in the horizontal position in group 3.

Experiment 2: In vivo feasibility test of the IFOT system

Experimental design

The experiments were approved by the Commission for the animal welfare of the University of Veterinary and Pharmaceutical Sciences Brno (UVPS Brno; Protocol number: 5/2017), complying with the ethical principles in animal research.

The animals used for the experiment were housed at the Ruminant and Swine Clinic of Faculty of Veterinary Medicine, UVPS Brno. The Holstein Friesian heifers were aged 15-17 months and had a body weight of $450 \mathrm{~kg}$. They were kept in a tie-stall, fed hay, concentrates, and a mineral supplement.

Firstly, the effect of injection of PBS into the preovulatory follicle (intrafollicular treatment, IFT) on the ovulation rate was tested. Heifers $(n=9)$ bearing a corpus luteum on the day of rectal examination were synchronized by i.m. administration of cloprostenol (500 $\mu \mathrm{g}$, Estrumate, Intervet, Boxmeer, Netherlands) followed by an i.m. injection of gonadorelin (GnRH, $50 \mu \mathrm{g}$, Gonavet, Veyx- Pharma, Schwarzenborn, Germany) $42 \mathrm{~h}$ later. Intrafollicular treatment was performed using the applicators filled with $200 \mu \mathrm{lof}$ PBS for IFT $20 \mathrm{~h}$ after the GnRH treatment.

In a further step, IFOT was performed. The synchronization scheme was used as previously described (Kassens et al. 2015). Briefly, heifers $(n=21)$ which had a corpus luteum on the day of rectal examination were synchronized by i.m. administration of cloprostenol $(500 \mu \mathrm{g})$ followed by an i.m. injection of $\mathrm{GnRH}(50 \mu \mathrm{g})$ $48 \mathrm{~h}$ later. A second cloprostenol treatment was performed 11 days later, followed by a second GnRH treatment after $42 \mathrm{~h}$. Artificial insemination was performed into the ipsilateral uterine horn $16 \mathrm{~h}$ after the second $\mathrm{GnRH}$ treatment. Intrafollicular oocyte transfer was performed 2-6 h later. Applicators filled with 110-120 $\mu 1$ PBS containing COCs were used for IFOT as described in Experiment 1. Applicators with loaded oocytes were positioned at an angle of 45 degrees with the needle downwards in the thermo-box at $30{ }^{\circ} \mathrm{C}$ and transported into the stall. The IFOTs were performed within 10-30 min. Follicular development was monitored by rectal ultrasonography at the time of the second cloprostenol and second GnRH treatment, immediately before IFOT, and to confirm the ovulation 6,24 , and $30 \mathrm{~h}$ after IFOT.

\section{Intrafollicular injection}

The presence and side of the preovulatory follicle was checked by rectal ultrasonography. Only heifers bearing a follicle of a diameter of at least $10 \mathrm{~mm}$ were used for IFT/IFOT. During the procedure, the heifers were kept in a cattle crush to restrict lateral movement during the procedures.

Heifers were sedated with detomidine hydrochloride i.v. (1.5 mg in total; Domidin $10 \mathrm{mg} / \mathrm{ml} \mathrm{Dechra,}$ Amsterdam, Netherlands) and epidural anaesthesia was performed (4 $\mathrm{ml}$ in total; lidocaine 2\%, Fatro, Ozzano, Italy). The tail was fixed, the rectum emptied, and the perineal region was cleaned and disinfected. Ultrasound jelly was put on the front part of the probe holder and covered by a latex probe protection. The holder with the fixed guide tube, protruding from the front part, was covered by a rectal glove for better hygiene of the IFOT. The holder was inserted into the vagina and placed dorso-cranially. The ovary bearing the preovulatory follicle was presented in front of the transducer by rectal manipulation. Rotation of the ovary was performed in a way that ensures IFOT through the ovarian stroma before reaching the follicular cavity to prevent leakage of the follicular fluid. The pre-warmed injector with attached applicator was inserted into the guide tube of the holder. The needle 
was carefully but vigorously inserted through the vaginal wall and peritoneum into the follicle cavity. When the tip of the needle reached the centre of the follicle antrum, the operator slowly moved the guide pin and pulled the rod. A distinct echogenic swirl of the follicle content was observed when the content of the applicator was expelled into the follicle. The needle was withdrawn from the follicle immediately after IFOT and the follicle stability was checked on the ultrasound screen. The applicator was flushed by PBS to the Petri dish and checked for remaining COCs under the stereomicroscope.

Embryo collection was done 7-8 days after IFOT by the usual flushing of the uterus in 15 heifers and by endoscopic flushing of the oviduct in 1 heifer. Upon the flushing completion, the animals were treated by cloprostenol i.m. $(500 \mu \mathrm{g})$.

The following variables were included in the evaluation of the success rate of individual IFOT sessions: the swirling of the follicle antrum during IFOT and unchanged follicle appearance after the injection; the ovulation rate of the injected follicle; the total number of recovered objects (unfertilized oocytes and embryos); and the number of recovered embryos.

\section{Statistical analysis}

With respect to the sample size in both experiments, statistical evaluation was performed only in Experiment 1. Experiment 2 concerned only nine heifers; therefore a complete description of experimental results is provided. Results of Experiment 1 were analysed using Pearson's $\chi^{2}$-test of independence used for comparison of ratios in considered groups. The test revealed significant differences on one per cent and one per mil levels as summarized in Tables 1 and 2, and was performed using R statistical software (R Core Team 2017).

\section{Results}

\section{Experiment 1: IFOT instruments feasibility test in the lab}

The impact of the length of the plastic collector in the applicator to the number of recovered COCs during the experiment was significant (Table 1). The number of oocytes recovered was significantly higher when using an $18 \mathrm{~mm}$ collector in comparison to one of $15 \mathrm{~mm}(P<0.001)$.

Table 1. Oocyte recovery rate in vitro using a different length of straw in the applicator.

\begin{tabular}{lccc}
\hline Straw length & Aspirated oocytes & Recovered oocytes & $\%$ \\
\hline $15 \mathrm{~mm}$ & 197 & $104^{\mathrm{a}}$ & 52.8 \\
$18 \mathrm{~mm}$ & 598 & $555^{\mathrm{b} * * *}$ & 92.8 \\
\hline
\end{tabular}

a,b within the column: values with different superscripts are significantly different

$* * * P<0.001$

The impact of the positioning of the applicator and the straw length on the number of oocytes recovered is summarized in Table 2 . The highest number of oocytes recovered was observed after the use of an $18 \mathrm{~mm}$ straw and a downward positioned applicator $(P<0.01)$.

Table 2. Oocyte recovery rate in vitro using different lengths of straw in the applicator and different positioning of the applicator.

\begin{tabular}{llccc}
\hline Straw length & Needle position & Stored & $\begin{array}{c}\text { Number of oocytes } \\
\text { Recovered }\end{array}$ & $\%$ \\
\hline $15 \mathrm{~mm}$ & Horizontal & 107 & $46^{\mathrm{a}}$ & 43.0 \\
& Downward & 90 & $58^{\mathrm{b} * *}$ & 64.4 \\
& Horizontal & 198 & $177^{\mathrm{c}}$ & 89.4 \\
$18 \mathrm{~mm}$ & Downward & 200 & $195^{\mathrm{d} * *}$ & 97.5 \\
& Upward & 200 & $183^{\mathrm{c}}$ & 91.5 \\
\hline
\end{tabular}

a,b,c,d within the column: values with different superscripts are significantly different

$* * P<0.01$ 


\section{Experiment 2: IFOT instruments feasibility test in vivo}

The ovulation rate

Intrafollicular injection of $0.2 \mathrm{ml}$ of PBS into the preovulatory follicle was performed in 9 heifers. Ovulation within $24 \mathrm{~h}$ after IFT occurred in 8 animals (88.9\%). The IFOT was performed in 21 heifers. Ovulation within $24 \mathrm{~h}$ after IFOT occurred in 18 animals $(85.7 \%)$. The total ovulation rate was $26 / 30(86.7 \%)$.

All intrafollicular injections performed in vivo were evaluated as successful (30/30, $100 \%$ ), due to the visible swirling motion of the follicular fluid at the time of the injection. No change in the size of the follicle immediately after injection was observed.

\section{Embryo recovery rate}

Embryo collection was done in 18 heifers, in which the ovulation of injected follicles had been observed. Embryos were flushed from the uterus in 15 heifers 7 days after IFOT; transvaginal endoscopy flushing of the oviduct was performed in 1 heifer 2 days after IFOT. Embryo collection was not successful in 2 heifers (impassable cervix, disturbed oviduct). In total, 316 oocytes were injected into the preovulatory follicles during 16 IFOT sessions (15-24 oocytes, an average of 19.7), finished by embryo collection The total number of recovered objects was $71(22.5 \%), 39$ unfertilized oocytes and 32 embryos $(10.1 \%)$.

The number of recovered objects varied from 0 to 18 . The recovery rate of 5-18 objects occurred on 6 occasions. Recovery rate higher than $50 \%$ of injected COCs (11-18) was observed in 4 events.

Embryos were recovered in 4 sessions $(25 \%)$, the number of recovered embryos in individual sessions differed: 5, 6, 6, and 16. The most successful embryo flushing $(n=16)$ was performed using transvaginal endoscopy. All calculations of recovered structures considered the recipient's own oocyte/embryo.

\section{Discussion}

We developed and verified the functionality of a new apparatus that allows aspiration of oocytes in laboratory conditions, their transport to the stall to recipients and the performance of the IFOT procedure by one operator with minimal assistance of a second person required to load the device in field conditions.

The IFOT procedure is technically challenging because the injection into the preovulatory follicle must not interfere with ovulation. There are two important requirements: the follicle injury as well as the volume of fluid applied must be kept to a minimum. The instruments for IFOT described in the literature have been based on a modified OPU device of similar construction (Kassens et al. 2015; Sprícigo et al. 2016) equipped with a different aspirating/injecting tubing system. The first IFOT set described (Kassens et al. 2015) uses $26 \mathrm{G}$ disposable needles $(0.45 \times 22 \mathrm{~mm})$ and PBS medium at a volume of $200 \mu$ l. The source of negative/positive pressure is a laboratory pipette attached to the tubing system. The second IFOT set described (Sprícigo et al. 2016) uses 27G disposable needles ( $0.4 \mathrm{~mm}$, length not specified) and follicular fluid sourced from abattoir ovaries at a volume of $60 \mu$ l. The source of negative/positive pressure is an insulin syringe attached to the tubing system.

The features of our system are similar. We use $25 \mathrm{G}$ disposable needles $(0.50 \times 40 \mathrm{~mm})$ and PBS at a volume of 150-200 $\mu$. The $25 \mathrm{G}$ needles are $0.05-0.01 \mathrm{~mm}$ thicker than previously referred, however, the difference is not important considering the follicle size (diameter of at least $10 \mathrm{~mm}$ ). The main advantage is the length of these needles because sometimes manipulation of an ovary bearing preovulatory follicle is difficult. A precision screw of the aspirator enables the plastic collector and needle to be filled with medium containing oocytes very slowly and at a small volume. This should minimize the change 
of follicular microenvironment by the injection. Phosphate buffered saline was chosen as an oocyte-carrying medium, because the use of follicular fluid from abattoir ovaries is not recommendable due to reasons of biosecurity.

In the first experiment, we verified the applicability of the tools under laboratory conditions. The aim was to find out whether it is possible to load oocytes in minimal volume into the applicator (aspiration needle and plastic collector), and whether the oocytes actually leave the interior of the applicator after manipulation with the guide pin and pulling rod. The reason for testing the appropriate position of the applicator before injection was that the applicators with loaded oocytes must be transported from the aspiration site (laboratory) to the transfer site (examination room, stable) when performing IFOT. This requires time in the order of minutes, possibly even tens of minutes for the intended implementation of IFOT in the field. During this time, the oocytes move in the system due to gravity. In addition, similar instrument positions may occur during IFOT on a live animal, although only for the relatively short period of the injection itself. Therefore, positioning the applicator before IFOT is important.

In the first stage of verification, we proved significantly more successful COCs recovery rates when using the $18 \mathrm{~mm}$ long cut straws. The explanation is that there is probably no turbulence in the space between the straw and the needle conus compared to the $15 \mathrm{~mm}$ long straw. The highest COCs recovery rates $(97.5 \%)$ were observed when the applicator was positioned with the needle downwards, compared to the other two positions $(89.4 \%$ and $91.5 \%$ ) (Tables 1,2). These conclusions confirmed the effect of applicator positioning before IFOT on the rate of recovered COCs. This implies that it is always necessary to maintain the position of the injection set before the actual IFOT in the needle-down position, where the aspirated oocytes sediment into one cluster. Oocytes behave as a single unit due to the adhesion of cumulus cells and this phenomenon allows most oocytes to be expelled at once.

In the second experiment we tested the instruments for IFOT in vivo. We found that the injector with an inserted applicator makes it possible to perform IFOT using modified basic equipment (probe holder, working tube) for OPU. All intrafollicular injections performed in vivo were evaluated as successful $(30 / 30,100 \%)$, since all injections clearly showed follicular fluid turbulence, the follicles retained their original shape and persisted in ovaries until ovulation. The ovulation rate of our treated follicles did not differ much $(85.7 \%)$ from the ovulation rate of commonly induced heat. This suggests that the effect of a properly performed IFOT on ovulation is likely to be negligible, but a higher number of trials is required to confirm this assumption definitively.

The numbers and quality of recovered objects (embryos/oocytes) collected from the uterus 7 days after IFOT were very variable. In total, $23.1 \%$ of the objects were obtained, which is slightly less than in the available literature, where $35.2 \%, 21.3 \%$, and $38.5 \%$ were reported (Kassens et al. 2015; Sprícigo et al. 2016; Hoelker et al. 2017). The average number of embryos obtained was $10.8 \%$. This figure does not differ much from the data in the available literature where $8.4 \%, 5.5 \%$, and $17.3 \%$ of the recovered embryos are reported (Kassens et al. 2015; Sprícigo et al. 2016; Hoelker et al. 2017). However, our results come from a low number of sessions, and therefore the best result achieved by endoscopic fallopian tube collection has certainly positively distorted the overall average.

The individuality of the animal appears to influence the results. Some of the animals used in the experiments were heifers that had been signed off and this could have had a negative effect on the results (ovulation rate, cervix). Therefore, further experiments focused on the quality of the recipient animal, precise synchronization, quality of follicle and timing of ovulation are necessary to improve the final outcome of the technique.

In conclusion, the study clearly proved the feasibility of the new instruments for IFOT in field conditions. The main perspective appears to be the connection with OPU technology 
when collected oocytes could be easily loaded and transferred to another farm and, without further manipulation, immediately injected into the preovulatory follicles of recipients. In the future, after further elaboration, the technique could be widely used in research as well as for routine embryo production.

\section{Acknowledgements}

The study was supported by Internal Grant Agency (119/2017/FVL) of UVPS Brno.

The authors thank U. Besenfelder and V. Havlicek (Reproduction Centre - Wieselburg, University of Veterinary Medicine, Vienna, Austria) for their support during the experiments and for endoscopic embryo collection.

\section{References}

Besenfelder U, Havlicek V, Kuzmany A, Brem G 2010: Endoscopic approaches to manage in vitro and in vivo embryo development: Use of the bovine oviduct. Theriogenology 73: 768-776

Findlay JK, Holland MK, Wong BBM 2019: Reproductive science and the future of the planet. Reproduction 158: R91-R96

Hoelker M, Kassens A, Salilew-Wondim D, Sieme H, Wrenzycki C, Tesfaye D, Neuhoff Ch, Schellander K, Held-Hoelker E 2017: Birth of healthy calves after intra-follicular transfer (IFOT) of slaughterhouse derived immature bovine oocytes. Theriogenology 97: 41-44

Kasinathan P, Wei H, Xiang T, Molina JA, Metzger J, Broek D, Kasinathan S, Faber DC, Allan MF 2015: Acceleration of genetic gain in cattle by reduction of generation interval. Sci Rep 5: 5-8

Kassens A, Held E, Salilew-Wondim D, Sieme H, Wrenzycki C, Tesfaye D, Schellander K, Hoelker M 2015: Intrafollicular oocyte transfer (IFOT) of abattoir-derived and in vitro-matured oocytes results in viable blastocysts and birth of healthy calves. Biol Reprod 92: 1-2

Landry DA, Bellefleur AM, Labrecque R, Grand FX, Vigneault C, Blondin P, Sirard MA 2016: Effect of cow age on the in vitro developmental competence of oocytes obtained after FSH stimulation and coasting treatments. Theriogenology 86: 1240-1246

Lonergan P, Fair T 2008: In vitro-produced bovine embryos-Dealing with the warts. Theriogenology 69: 17-22

Moreno D, Neira A, Dubreil L, Liegeois L, Destrumelle S, Michaud S, Thorin C, Briand-Amirat L, Benchasroff D, Tainturier D 2015: In vitro bovine embryo production in a synthetic medium: Embryo development, cryosurvival, and establishment of pregnancy. Theriogenology 84: 1053-1060

O'Doherty AM, McGettigan P, Irwin RE, Magee DA, Gagne D, Fournier E, Al-Naib A, Sirard M-A, Walsh CP, Robert C, Fair T 2018: Intragenic sequences in the trophectoderm harbour the greatest proportion of methylation errors in day 17 bovine conceptuses generated using assisted reproductive technologies. BMC Genomics, 19: 1-15

Pieterse M, Kappen K, Kruip T, Taverne M 1988: Aspiration of bovine oocytes during transvaginal ultrasound scanning of the ovaries. Theriogenology 30: 751-762

R Core Team 2017: R: A language and environment for statistical computing. R Foundation for Statistical Computing, Vienna, Austria

Sprícigo JFW, Sena Netto SB, Muterlle CV, Rodrigues S de AD, Leme LO, Guimarães AL, Costa Caixeta FM, Machain Franco M, Pivato I, Dode MAN 2016: Intrafollicular transfer of fresh and vitrified immature bovine oocytes. Theriogenology 86: 2054-2062

Velazquez MA, Kues WA, Niemann H 2014: Biomedical applications of ovarian transvaginal ultrasonography in cattle. Anim Biotechnol 25: 266-293 
Plate I

Andrlikova M. et al.: Intrafollicular oocyte... pp. 11-17

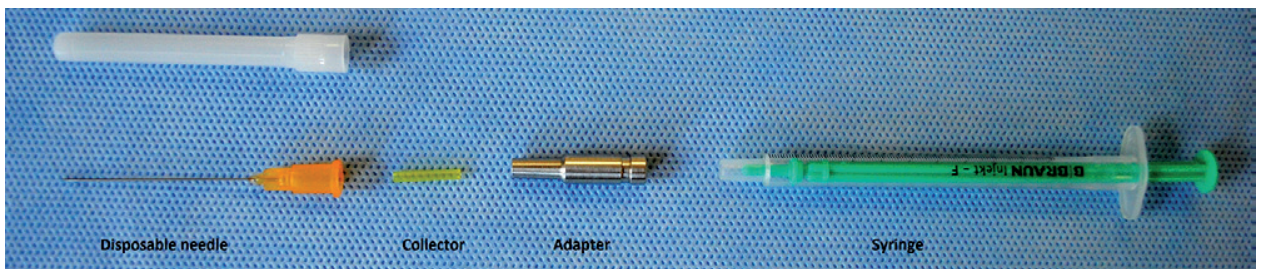

Fig. 1. Dismantled applicator

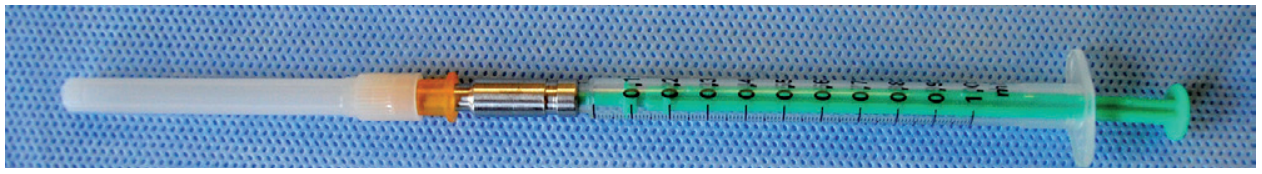

Fig. 2. Assembled applicator

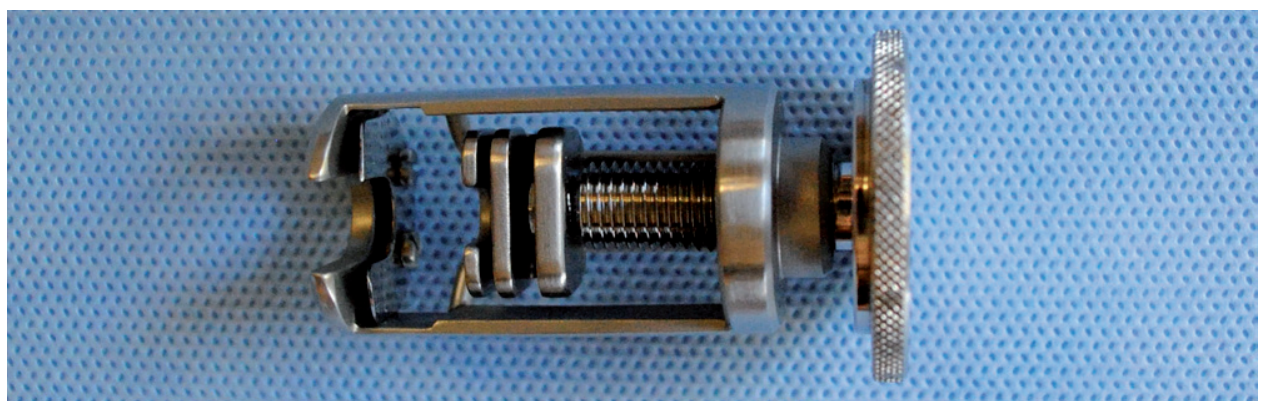

Fig. 3. Aspirator

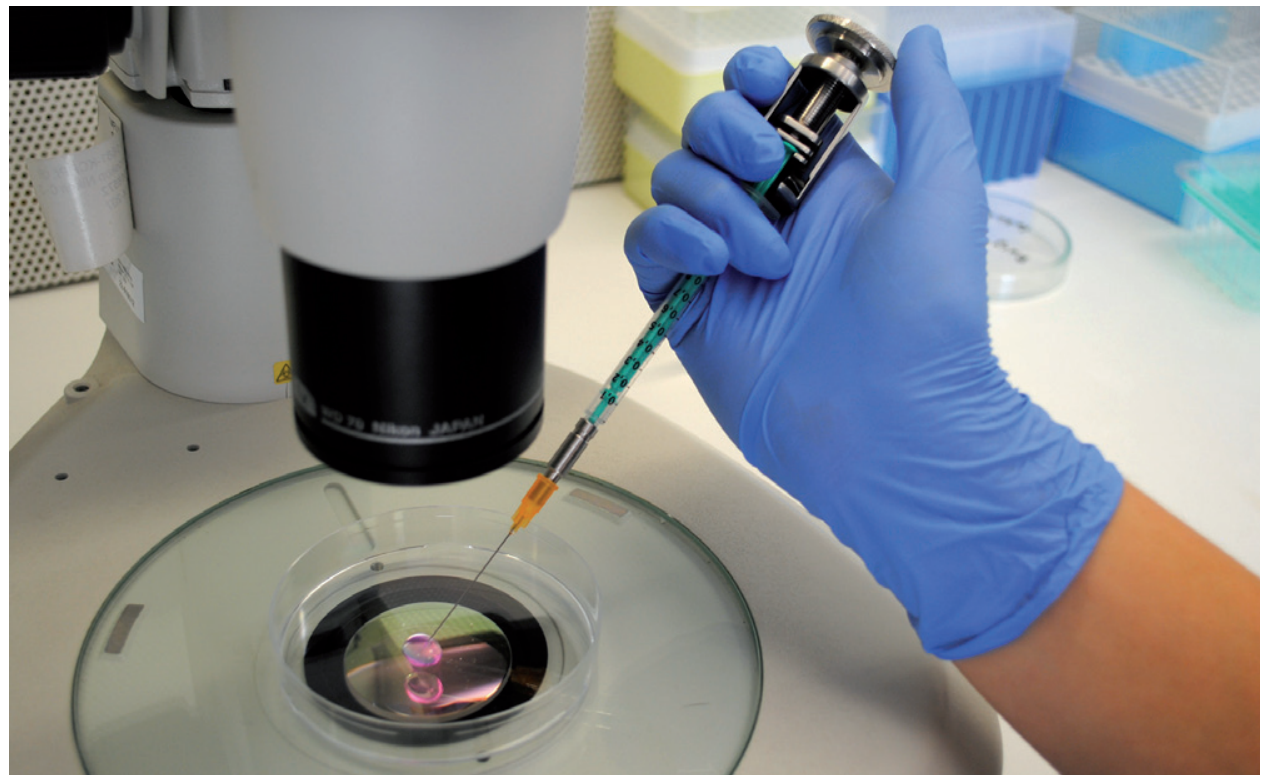

Fig. 4. The loading of oocytes (2) 
Plate II

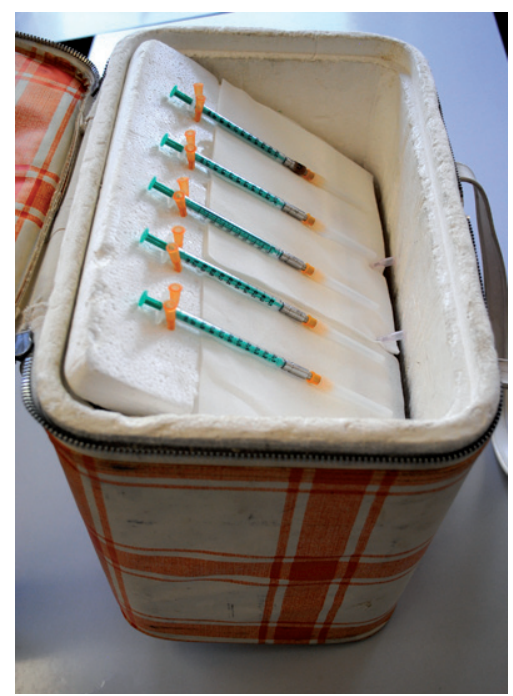

Fig. 5. Applicators prepared for transport

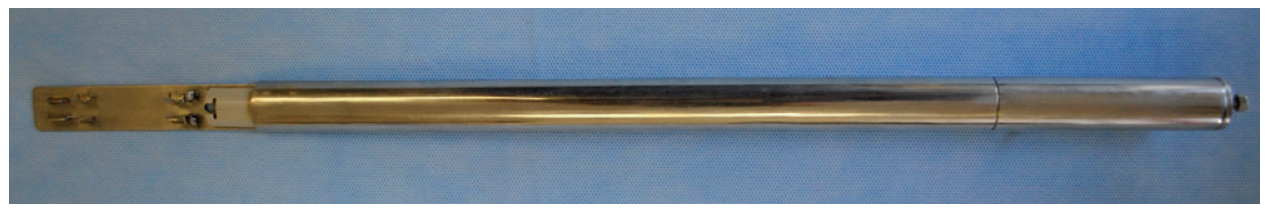

Fig. 6. Injector

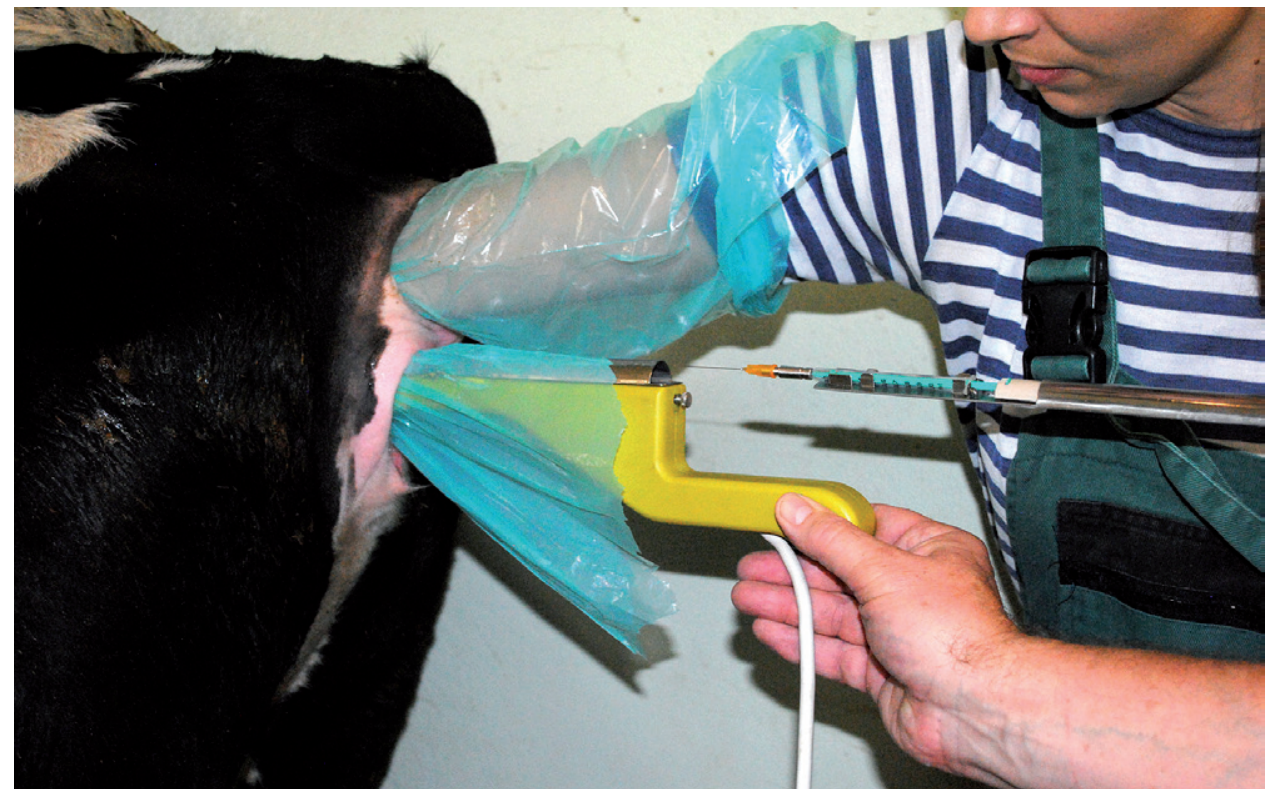

Fig. 7. Injector inserted into the guide tube 
Plate III

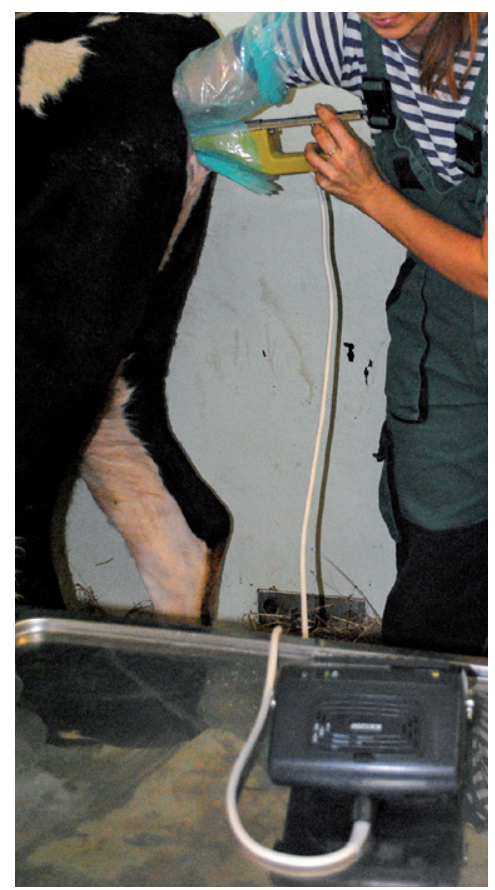

Fig. 8. IFOT 